\title{
O PROBLEMA DAS DOENÇAS CRONICAS E DEGENERATIVAS E DOS ACIDENTES NAS ÁREAS URBANIZADAS DA AMÉRICA LATINA
}

LAURENTI, R. - O problema das doenças crônicas e degenerativas e dos acidentes nas áreas urbanizadas da América Latina. Rev. Saúde públ., S. Paulo, 9:239-48, 1975.

REsumo: A rápida urbanização que vêm sofrendo as cidades latino-americanas tem apresentado alguns problemas de saude para as suas populações. As doenças crônicas $e$ degenerativas $e$ os acidentes, principalmente os de trânsito $e$ os de trabalho, têm tido nessas áreas uma evolução ascendente quanto à incidência, medida principalmente pela mortalidade. Dentre as doenças crônicas, as cardiovasculares e os tumores malignos apresentam coeficientes de mortalidade por vezes superiores aos observados em cidades de paises desenvolvidos. São discutidas também as incidências dos acidentes de trânsito $e$ os de trabalho, as quais são bastante superiores às verificadas nos paises desenvolvidos. Esses aspectos vêm cada vez mais constituindo problemas quanto à demanda de serviços, porêm é discutido o fato de que ainda não devem ser considerados prioritários, tendo-se em vista que, ao lado destes agravos a saúde, existem também, em altos niveis, as doenças infecciosas, a maioria das quais ja foram completamente dominadas em paises desenvolvidos. Assim, por exemplo, em São Paulo, ainda que seja grande a demanda de serviços para as doenças crônicas $e$ os acidentes, parte considerável dos gastos ainda estão voltados para as doencas infecciosas.

Unitermos: Urbanização. Saúde Pública, América Latina. Doenças crônicas e degenerativas. Acidentes.

O rápido crescimento de cidades em todo o mundo, particularmente em países em vias de desenvolvimento, vem criando problemas sociais e dentre esses aqueles relacionados à saúde dessas populações. A América Latina vem apresentando um grande processo de urbanização nas últimas décadas e segundo o 8. Relatório do Banco Interamericano de Desenvolvimento ${ }^{1}$ (BID), "O crescimen- to geral da população da América Latina é o mais alto entre as grandes regiōes do mundo desde 1920. Não é menos significativo, entretanto, que a taxa de crescimento da população urbana haja sido também a mais alta desde aquele mesmo ano, superada unicamente pela África no período 1950-1960".

Comparando-se as taxas de crescimento da população urbana e rural, verifica-

* Da Disciplina de Estatistica Vital, Depto. de Epidemiologia, Faculdade de Saúde Pública da USP - Av. Dr. Arnaldo, 715 - São Paulo, SP - Brasil 
I.ATRENTI, R. - O problema das doencas cronicas e degenerativas o dos acidentes nas áreas urbanizadas da América Latina. Rev. Sañde públ:, S. Paulo, 9:239-48, 1975.

se que a primeira. de um modo geral. é maior. As grandes concentrações urbanas representam. nos países latino-americanos. percentagem elevada da população total. ('omo pode ser verificado na Tabela 1.

() Brasil em 1960 apresentava 14 cidades com população entre 100 e $250 \mathrm{mil}$ habitantes. passando a $32 \mathrm{em} \mathrm{1970;} \mathrm{com}$ população superior a $250 \mathrm{mil}$ hahitantes passou de 10 para 15 cidades. Dentre as grandes cidades do Brasil, São Paulo é um exemplo típico de urbanização rápida. pois em 1940 possuia 1.314 .952 hahitantes passando em 1950 a 2.198 .096 , em 1960 a 3.675.602 e em 1970 atinge

5.931.595 hahitantes. A estimativa para 1975 é superior a 7.000.000, sendo que a área metropolitana (Grande São Paulo) alcançará. aproximadamente, $11 \mathrm{mi}-$ lhões de hahitantes.

Este processo de urbanização tem trazido várias conseqüências criando problemas para a saúde coletiva e para os serviços de assistência médica. Talvez os maiores problemas sejam ainda aqueles conseqüentes à falta de saneamento básico e a incidência de algumas doenças infecciosas. Por sua vez, as doenças crônicas e degenerativas vêm apresentando um aumento, assim como os acidentes, quer os de trânsito quer aqueles de trabalho.

\section{AS DOENCAS CRONICAS E DEGENERATIVAS}

Essas doenças, entre as quais serão citadas, como exemplo, as cardiovasculares e os tumores malígnos, vêm assumindo cada vez mais um importante papel na morbidade e na mortalidade em áreas de crescente urbanização na América Latina. Em muitas delas, os coeficientes de mortalidade apresentam-se bastante próximos $e$ inclusive em algumas áreas igualam ou superam aqueles observados em cidades de países desenvolvidos, europeus ou norte-americanos.

Lima pesquisa levada a efeito em 10 cidades latino-americanas, uma norte-ame-

TABELA I

Percentagem que representa a população de 5 malores cidades em relação ao total da população do pais - América Latina 1950,1960 e 1970

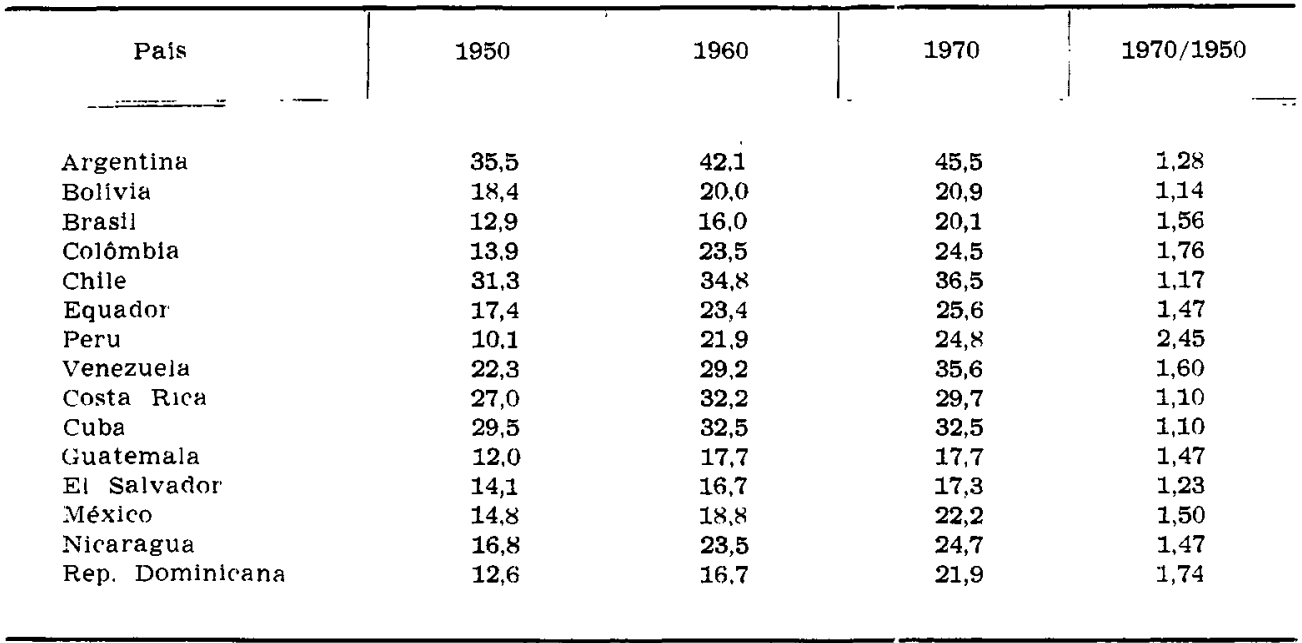

Fonte: Notas sobre la Economla y el Desarrollo de America Latina (Comissão Econômica para a América Latina - CEPAL,. Santiago. Chile, no 85, Out. 1971. 
I.ALRENTI. R. - O problema das doencas cronlcas e degenenativas c dos acldentes nas áreas urbanıadas da Amérlea Latina. Rev. Sahte pibl. S Paulo, 9:239-4x, 1975.

ricana e outra inglesa mostrou claramen$t^{2}$ a importância das doenças crônicas e degenerativas para as cidades da Améri4a Latina". Eser estudo analisou a mortalidade de adultos de 15 a 74 anos. atravé. de uma investigação minuciosa de rada caso por meio de entrevistas domi-iliares a aos médicos. hospitais. etc. que atenderam os falecidos. podendo-se assim (aracterizar perfeitamente a mortalidade por causas. mo todas as áreas. sem ha-ear-se em estatisticas oficiais. nen sempret fidedignas.

A Tabela 2 mostra. para ambos os sesos. os cueficientes de mortalidade e a percentagem sobre o total de mortes de 15 a 74 anos nas 12 cidades. para as doenças cardiorasculares. tumores malíg$n o s e$ para refito de comparação. as doenças infecciosas e parasitárias.

$O$ s coeficientes de mortalidade por doenças rardiovasulares para o sexo masculino. em todas as áreas latino-americanas estudadas. foram inferiores aos obserrados em Bristol e San Francisco.

Vo sexo feminino somente três áreas 1 Guatemala. La Plata e Lima) apresenlaram coeficientes inferiores aos de Bristol e San Francisco. Em lodas as demais. os coeficientes foram maiores. atingindo em Bogotá quase o dobro do rerificado nas duas cidades de língua inglesa.

A análise dos diferentes tipos de doencas cardiovasculares mostrou para as cidade: latino-americana: a importância da dotnça isquêmica do coração. no sexo masculino. " das lesoes rasculares que aftam o sistema nerroso central. para o -exo feminino. Em todas as áreas latinu-americanas texceto Caracas) no sexo feminino. foi maior a mortalidade por lesoes rasculares que afelam o sistema neroso central. em relação à doenca isquêmica do coração. Em Brislol e Saı Francion verificon-se o contrário".

Ainda que a análise dos diferentes tjyos de doenças cardiovasculares tenha mu-trado que cada área apresenta uma característica própria. é importante destacar que. em conjunto. elas representam uma importante causa de morte em áreas urhanizadas da América Latina. da mesma maneira que em cidades de países desenvolvidos. Vestes rem sendo obserrado um aumento crescente da incidência das doenças caidiorasculares. Assim. por exemplo. nos Estados T-nidos. em 1900. 20\% das moites foram consequentes a essas causas, alıançando no início da década de 60 . valores ao redor de 55\%; Isso foi derido. em grande parte. aos progressos conseguidos na redução das doenças infecciosas \& da desnutrição que. diminuindo a mortalidade. aumentou a vida média. farorecendo o surgimento daquelas causas.

A análise da rolução no tempo deste grupo de causos nas áreas urbanizadas da América Latina nem sempre é fácil de realizar devido à dificuldade de se conseguir dados. Será apresentado o que vem ocorrendo na cidade de São Paulo que é. como se sabe. uma das áreas de mais rápida urbanização dentre os países latino-americanos.

Em São Panlo. no hiênio $1940 / 11$ a mortalidade por doenças cardiovasculares era de 227.3 por 100.000 habitantes. correspondendo a ]6.8\%; de todas as mortes - se elevou para 262.6. passando a representar $30.6 \% \mathrm{C}$ do total de óbitos. 110 liênio 1968 1969. O coeficiente de mortalidade aumentou 1.2 vezes enquanto que a proporção de mortes por essas causas aumentou 1.8 vezes.

Os rlados apresentados para dez cidades latino-americanas * a evolução da mortalidade por doenças cardiorasculares rm uma delas. mostran que essas doenças se apresentaram de maneira destacada e que nas últimas décadas sua importância vem crescendo. o que certamente indica um aumento da morhidade. Esse quadro vem dando origem a alqums proli]emas o que erá comentado mais adiante. juntamente com on Lumores malignos e os acidentes. 
LAURENTI, R. - o problema das doenças crônicas e degenerativas e dos acidentes nas áreas urbanizadas da América Latina. Rev. Saúde públ., S. Paulo, 9:239-48, 1975.

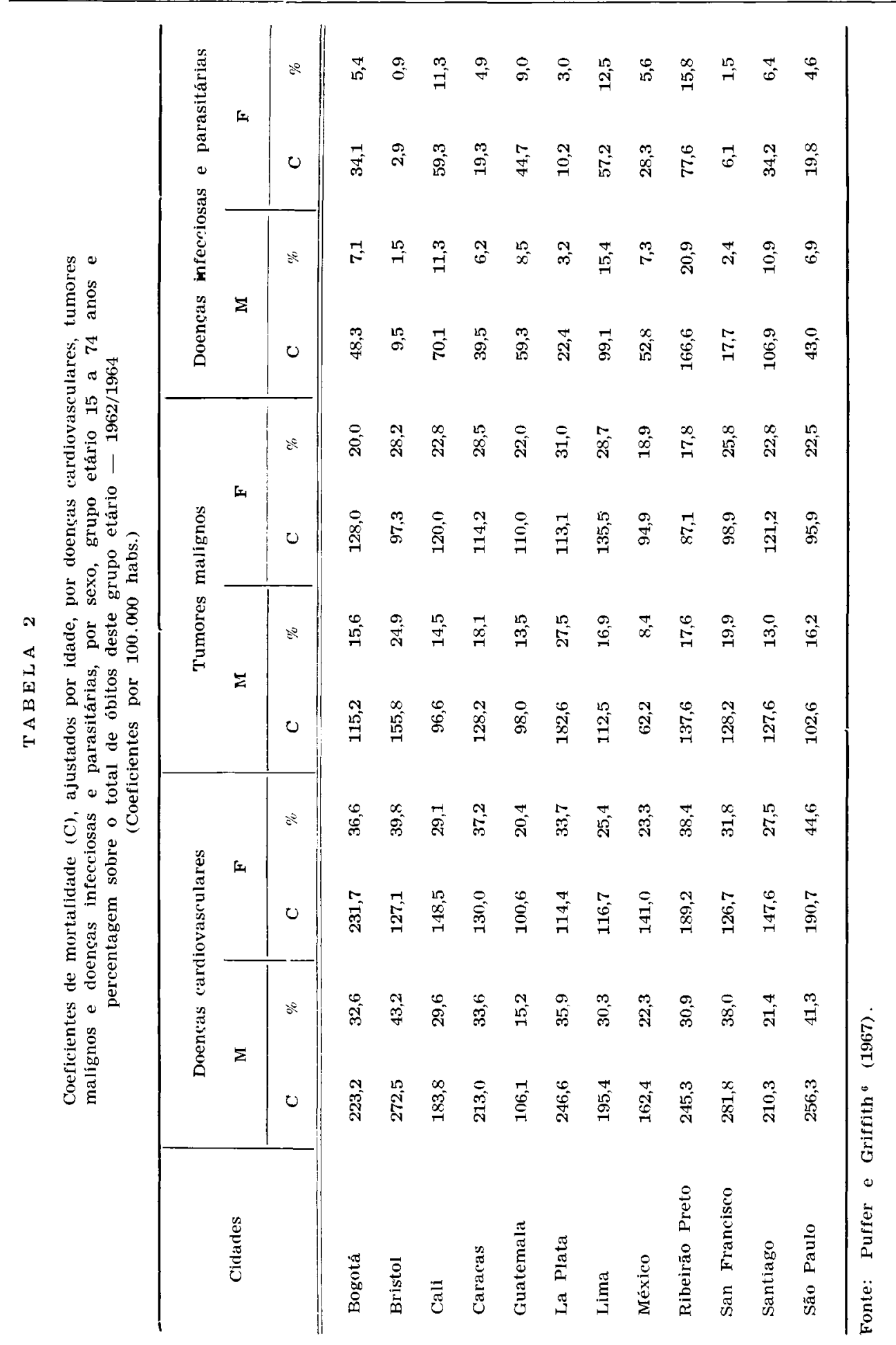


LAURENTI, R. - O problema das doencas crônicas e degenerativas e dos acidentes nas áreas urbanizadas da América Latina. Rev. Saúde pübl., S. Paulo, 9:239-48, 1975.

Quanto aos tumores malígnos, pode-se apreciar na Tabela 2 os valores dos coeficientes de mortalidade nas dez cidades latino-americanas e compará-los com os observados em Bristol e San Francisco. Naquelas cidades os coeficientes são muito semelhantes aos observados nas cidades inglesa e norte-americana, sendo que algumas delas os apresentam maiores, o que é mais evidente no sexo feminino.

E importante ressaltar o fato de que cada área apresenta características próprias no que diz respeito a tipos de tumores malígnos mais freqüentes, o que traduziria aspectos epidemiológicos específicos que precisam ser levados em conta. Assim, o estudo do qual são tiradas essas informaçōes ${ }^{6}$ mostrou que, no que diz respeito ao câncer de estômago, Bogotá apresenta um nível muito alto de mortalidade que é, por exemplo, 6 vezes superior ao observado na cidade do México e 1,4 vezes maior que na cidade da Guatemala que apresentou o segundo coeficiente de mortalidade (Tabela 3 ).

\section{T A B E L A 3}

Nivel dos coeficientes de mortalidade, ajustados por idade, devido a tumor maligno do estômago no grupo etário 15 a 74 anos em 10 cidades latino-americanas, uma norte-americana e outra inglesa - 1962/1964

(Coeficiente por 100.000 habitantes)

\begin{tabular}{l|l|c}
\hline $\begin{array}{c}\text { Nivel } \\
\text { de } \\
\text { mortalidade }\end{array}$ & Cidades & $\begin{array}{c}\text { Coef. de } \\
\text { mortali- } \\
\text { dade }\end{array}$ \\
\hline \hline Muito alto & Bogotá & 41,3 \\
& Guatemala & $\mathbf{2 8 , 8}$ \\
Alto & Rib. Preto & $\mathbf{2 8 , 6}$ \\
& Santiago & 27,6 \\
Lima & 25,4 \\
Médio & Cali & 23,4 \\
& S. Paulo & 21,2 \\
Baixo & Caracas & $\mathbf{1 9 , 7}$ \\
Muito baixo & La Plata & $\mathbf{1 5 , 7}$ \\
& Bristol & $\mathbf{1 3 , 1}$ \\
& S. Francisco & $\mathbf{8 , 1}$ \\
\hline
\end{tabular}

Fonte: Puffer e Griffith ${ }^{6}$
Também o câncer de pulmão apresentou uma grande variação quanto aos níveis de mortalidade nas diferentes cidades latino-americanas, sendo que em uma delas (La Plata) o coeficiente para o sexo masculino foi, praticamente, igual ao observado na área inglesa (Bristol) e superior ao da área norte-americana (San Francisco). La Plata apresentou, para o sexo masculino, uma mortalidade bastante superior àquela observada nas outras áreas latino-americanas (de 2,4 a 10 vezes superior).

Ainda quanto aos tumores malígnos, um aspecto interessante é o que diz respeito àqueles de mama e colo uterino. Sabe-se que nas áreas mais desenvolvidas, comc Estados Unidos e países europeus, são mais freqüentes os tumores de mama em relação aos do colo uterino. Fato inverso observa-se nas áreas sub-desenvolvidas.

As áreas urbanizadas latino-americanas, ou pelo menos a maioria delas, ainda apresentam um predomínio do câncer de colo uterino em relação ao de mama. Comparando-se as 12 cidades que participaram do estudo de mortalidade ${ }^{6}$, verificou-se que Bristol, San Francisco e La Plata apresentaram os menores coeficientes de mortalidade por câncer de colo uterino e os maiores por câncer de mama. Das 10 cidades latino-americanas, somente La Plata, São Paulo e Ribeirão Preto apresentaram maior mortalidade por câncer de mama em relação aos de colo uterino, porém, apenas em La Plata os niveis eram semelhantes aos observados em Bristol $€$ San Francisco.

Independentemente dos tipos de tumor maligno, considerando-os em conjunto, tem sido verificado nas últimas décadas um aumento da mortalidade nas áreas urbanizadas da América Latina, o que, pode-se admitir, seja um reflexo do aumento da incidência. Podemos citar, como exemplo, o que vem sendo observado em São Paulo, onde o coeficiente de mortalidade aumentou, aproximadamente, $20 \%$ 
IALRENTI. R. - O problema das doencas crônicas e degenerativas e dos acidentes nas áreas urbanizadas da América Latina. Rev. Saúle publ., S Paulo, 9:239-4^, 1975.

de $1940 / 41$ a $1968 / 69$. sendo que a mortalidade proporcional em relação a todas as outras causas aumentou $80 \%$.

\section{ACIDENTES DE TRÁNSITO E DE TRABALHO}

Fsses agravos à saúde e à vida vêm. "adia vez mais. constituindo sérios problemas na América Latina. O número de vítimas e. particularmente, de mortes representa um importante contingente na morbidade e morlalidade geral. sendo que o prohlema se torna mais grave se se levar em conta que a população mais afetada é aquela incluída no grupo economicamente ativo.

Quanto aos acidentes de trârisito, a comparação feita entre o que ocorre nos ETA e em onze países latino-americanos, mostrou que pxiste maior proporção de feridos por veículos na América Latina $(325 / 10.000$ veículos) que naquele país (196/10.000 veículos) ${ }^{*}$. Isso poderia ser explicado pela menor proteção aos usuários das vias públicas e também pelo haixo nivel de instrução dos motoristas c pedestres.

Os novos habitantes que vão chegando às cidades. muitas vezes em rítmo acelerado. encontram maiores facilidades de emprego e, na maioria das vezes. salários mais elevados que os percebidos nas áreas de origem. A aquisição de certos bens de consumo está aumentando por parte dessas populaçôes e entre esses está o automóvel. Por outro lado. na maioria das áreas Jatino-americanas não existe uma "tratlicão automohilística" verificando-se um baixo nivel de educação para o trânsito. o que é responsável. em grande parte, pelas altas freqüências de acidenles. vitimas a mortes.

Tomando-se como exemplo, mais uma vez. a cidade de São Paulo. pode-se medir a importàn ja do problema. através das magnitudes dos coeficientes de mortalidade por acidentes de veículo a motor:
Coeficientes

Anos

(por 100.000 habs.)

$\begin{array}{cr}1940 / 41 & 8.7 \\ 1950 / 51 & 12.2 \\ 1960 / 61 & 15.7 \\ 1970 / 71 & 33.6 \\ 1973 & 46.7\end{array}$

Como se pode verificar, o risco de morrer por acidente de trânsito de 1940/41 para 1973 aumentou 5.4 vezes. No biênio $1940 / 41$ morreram 236 pessoas. sendo que no biênjo $1970 / 71$ esse número se elevou a 5.964. Em 1970 um estudo efetuado em São Paulo mostrou que $65 \%$ dos falecidos por acidentes de trânsito haviam recebido atendimento hospitalar após o acidente ${ }^{4}$. Isso representa uma grande demanda de leitos hospitalares, principalmente levando-se em conta o número de vítimas que não morreram, estimandose que proporção apreciável delas receberam tratamento médico/hospitalar, sendo que essa demanda cresce de ano para ano. Assim, em São Paulo em 1970 registraram-se 19.348 feridos por acidentes de veículo a motor, número esse que se elevou a 36.474 em 1973. portanto, um aumento de $88 \%$.

Outro tipo de acidente que merece ser considerado como um problema de saúde relacionado a áreas urbanizadas latino-americanas $\dot{c}$ o acidente de trahalho. Praticamente em todas as grandes cidades está se verifirando umı industrialização crescente e cada dia maior número de trabalhadores são necessários para as mais diferentes atividades. Estas vão desde as mais sofisticadas. que requerem pessoal especializado. até certos tipos de atividades em que o nível do pessoal exigido não requer nenhum grau de preparo. como por exemplo os operários da construção civil. Esse tipo de atividade requer grande número de trahalhadores. a a maioria deles são procedentes de zonas rurais ou the pequenas cidades.

Tem sido verificado que a frequêencia de acidentes de trahalho na América La- 
LAURENTI, R. - O problema das doenças cronicas e degenerativas e dos acidentes nas áreas urbanızadas da América Latina. Rev. Saíle pübl., S. Paulo, 9:239-48, 1975.

tina é grande quando comparada a países altamente industrializados. Isso se deve ao fato de que as medidas preventivas não são adotadas da maneira adequada ou, também possivelmente, porque o nivel de instrução do operário impede ou dificulta a aplicação delas.

No Brasil, segundo o Instituto Nacional de Previdência Social (INPS) vem aumentando o número e a proporção de acidentes de trabalho. Os gastos que isso provoca são muito grandes e em 1973 , eles corresponderam a $6 \%$ do orçamento da Nação e $1 \%$ do Produto Nacional Bruto. Para se ter uma idéia do número de acidentados em trabalho, de 1969 a 1972. no Brasil, alguns dados são apresentados na Tabela 4.

T A B E L A 4

Trabalhadores segurados pelo INPS, número e proporção de acidentes - Brasil, 1969 a 1972

\begin{tabular}{l|c|c|c}
\hline \multirow{2}{*}{ Ano } & $\begin{array}{c}\text { Trabalha- } \\
\text { dores } \\
\text { segurados } \\
\text { INPS }\end{array}$ & \multicolumn{2}{|c}{ Acidentes } \\
\hline & N.o & $\%$ \\
1969 & 7.268 .449 & 1.059 .296 & 14,5 \\
1970 & 7.284 .022 & 1.220 .111 & 16,7 \\
1971 & 7.553 .472 & 1.330 .523 & 17,6 \\
1972 & 7.773 .374 & 1.504 .723 & 19,3 \\
\hline
\end{tabular}

Fonte: Dados fornecidos pelo Instituto $\mathrm{Na}$ cional de Previdência Social (INPS)

Em países altamente industrializados, a freqüência de acidentes de trabalho varia em torno de 10 por 1 milhão de homens/ horas trabalhadas; em São Paulo alguns dados fornecidos pelo Serviço Social da Indústria (SESI) indicam que a freqüência atingiu, em 1971, 44 acidentes por 1 milhão de homens/horas trabalhadas, sendo que esse valor variou com o tipo de atividade industrial. Assim, é da ordem de 177 para os trabalhadores em construção civil. 84 para os metalúrgicos. 80 para os do setor gráfico. entre outros. e de 12 para os operários da indústria farmacêutica, 15 para o setor de perfumaria, 17 para a indústria química. sendo estes três últimos citados os mais baixos coeficientes de freqüência de acidentes que foram observados ${ }^{2}$.

Além dos aspectos acima referidos. quar.to às doenças crônicas e degenerativas e dos acidentes em áreas de rápida urbanização, outros poderiam ser comentados. Apenas serão mencionados os problemas ligados a vários tipos de poluição (do ar, sonora, entre outros) e o problema dos transtornos mentais. Vinogradov ${ }^{10}$ refere que, de cada 4 habitantes dos países ocidentais, um sofre distúrbio do sono e usa sonífero. Ainda que não se disponha de dados é bem possível que o meșmo ocorra nas cidades latino-americanas. $O$ mesmo autor faz referências ao alcoolismo crônico, que estaria aumentando nas cidades entre os indivíduos na idade produtiva.

\section{PROBLEMAS RELACIONADOS AO AUMENTO DAS DOENÇAS CRONICAS E DEGENERATI- VAS E DOS ACIDENTES}

Um aspecto importante relacionado ao aumento das doenças crônicas e aos acidentes é aquele referente à demanda de assistência médica. Realmente, cada vez mais é necessária maior quantidade de serviços especializados, tendo-se em vista aquele aumento.

Isso dá origem a um importante problema que é, talvez, o mais grave: ainda que nas áreas latino-americanas de rápida urbanização as doenças crônicas e os acidentes se apresentam com freqüência muitas vezes igual (às vezes até maior) à de áreas mais desenvolvidas. nelas ainda existem altos indices de doenças infecciosas. Apesar de que as necessidades quanto ao atendimento de doenças crônicas e acidentes sejam crescentes. ainda é necessário dispor de grande parte dos recursos para as doenças infecciosas. 
LAURENTI, R. - O problema das doencas crônlcas e degenerativas e dos acidentes nas áreas urbanizadas da América Latina. Rev. Saúde públ., S. Paulo, 9:239-48, 1975.

A Tabela 2 mostra que, mesmo para o grupo etário onde as doenças infecciosas não são tão freqüentes ( 15 a 74 anos), os coeficientes de mortalidade são altos quando comparados aos de áreas desenvolvidas. Em algumas capitais latino-americanas chegam a representar de 11 a $15 \%$ de todos os óbitos naquele grupo etário.

Seria interessante medir e comparar a demanda de atendimento médico para as doenças crônicas e acidentes, de um lado, e de outro, aquela para as doenças infecciosas. Uma avaliação mais ou menos grosseira disto poderia ser feita através dos resultados de uma pesquisa em que se analisaram as causas múltiplas de óbitos ocorridos em hospitais da cidade de São Paulo, onde para cada caso foi verificado o número de diagnósticos existentes e que inecessitaram algum tipo de recurso médico, independente de ser ou não a causa básica da morte ${ }^{5}$. $\mathrm{Na}$ amostra de 1.832 casos, de todas as idades, foram contados 5.385 diagnósticos e quanto a algumas doenças crônicas e acidentes (todos os tipos) verificou-se o seguinte: tumores malígnos, 321 diagnósticos (5,9\% do total de diagnósticos); diabetes, $77(1,4 \%)$; doenças cardiovasculares, $1.329(25,4 \%)$; transtornos mentais, $50(0,9 \%)$ e acidentes, $182(3,4 \%)$.

Observou-se então que aqueles grupos de causas acima citados foram responsáveis por $37,0 \%$ dos diagnósticos, recebendo os pacientes algum tipo de atendimento médico, independente de ter ou não outra doença, inclusive infecciosa.

Por outro lado, existiram 748 diagnósticos de doenças infecciosas $(13,8 \%)$ e $394(7,3 \%)$ de desnutrição (qualquer tipo, incluindo avitaminoses). Portanto esses dois grupos de causas contribuiram com $21,1 \%$ do total de diagnósticos que requereram cuidados médicos.

Ainda que o estudo supracitado se refira somente a causas de mortes ocorridas em hospitais é possivel ter-se uma idéia aproximada do tipo de demanda aos serviços médicos. Pode-se imaginar que um quadro semelhante ocorra com os casos internados e que não faleceram e também com os atendimentos em ambulatórios e outros serviços médicos.

Como se verifica, as doenças crônicas e os acidentes são responsáveis pela maior demanda de recursos médicos. Todavia, o grande problema é que parte importante dos recursos disponíveis ainda são necessários para as doenças infecciosas e desnutrição, condições praticamente inexistentes em áreas urbanizadas de países mais desenvolvidos.

A alta frequiência de doenças infecciosas nas áreas urbanas da América Latina se deve, principalmente, às precárias condições de saneamento básico, à desnutrição, e mesmo, para algumas áreas, à inexistência de bons programas de imunização. Em São Paulo, a principal causa de mortalidade infantil é a doença diarréica, responsável por $30,0 \%$ dos óbitos, e quanto à mortalidade de 1 a 4 anos, a principal causa é o sarampo (aproximadamente $20,0 \%$ ). 0 binômio infecçãodesnutrição é o grande responsável pelós altos níveis de mortalidade infantil e mortalidade de 1 a 4 anos, não só em São Paulo, mas em várias outras áreas urbanas da América Latina, como mostraram os resultados da "Investigação Interamericana de Mortalidade na Infância" "

Outro fato a ser destacado quanto às doenças infecciosas em áreas de grande urbanização é que os migrantes que aí chegam trazem consigo, muitas vezes, doenças infecciosas próprias de áreas rurais. Na cidade de São Paulo, o que não se inclui na área endêmica de doença de Chagas, verificou-se, em 1971, que 19,0 por mil indivíduos de 18 anos, convocados para o Serviço Militar, apresentavam a Reação de Machado-Guerreiro positiva e 2,4 por mil a apresentavam duvidosa ${ }^{3}$.

Do que foi exposto pode-se concluir que, provavelmente, a maioria das grandes cidades latino-americanas está em uma 
LAURENTI, R. - O problema das doenças cronicas e degenerativas a dos acidentes nas áreas urbanizadas da América Latina. Rev. Saúde públ., S. Paulo, 9:239-48, 1975.

situação bastante complexa no que se refere a problemas de saúde de suas populaçôes. Por um lado, as doenças crônicas e os acidentes se apresentam com freqüência alta e crescente; por outro lado estão ainda as doenças infecciosas, muitas das quais em niveis tão elevados quanto aqueles observados em zonas rurais. Somente quando se conseguir uma diminuição das doenças infecciosas, como ocorreu na América do Norte e Europa, poder-se-á situar as doenças crônicas e os acidentes como aspectos prioritários da saúde dessas populações.

Existe, porém, a problemática das doenças crônicas e dos acidentes e ela precisa ser levada em conta nas programaçōes de saúde. Além dos serviços médicos tipo curativo, deve-se pensar também em alguns aspectos preventivos tais como: educação sobre certos fatores de risco nas doenças cardiovasculares; no que diz respeito a neoplasias malígnas são importan- tes os aspectos preventivos e o diagnóstico precoce, principalmente para alguns tipos como o câncer de colo uterino, além de estudos epidemiológicos que possam caracterizar tumores mais predominantes em certas áreas. No que diz respeito aos acidentes de trânsito deve-se visar a educação do motorista e do pedestre, além da preconização de certas medidas de segurança.

Quanto aos acidentes de trabalho devem ser preconizadas as medidas que visam a prevenção, a fim de alcançar os mesmos níveis que os observados em áreas de grande tradição industrial. Essas medidas existem e devem ser postas em prática, ainda que o resultado irá depender, em grande parte, da melhoria do nível de instrução dos trabalhadores, visto que a maior parte dessas populações procede de áreas rurais e passa diretamente a atividades industriais dos grandes centros urbanos.

LAURENTI, R. - [The problem of chronic and degenerative diseases and of accidents in the urban areas of Latin America]. Rev. Saúde públ., S. Paulo, 9:239-48, 1975 .

Summary: Some health problems have been enhanced by the swift urbanization that Latin. America cities are going through at present. Chronic and degenerative diseases besides accidents, moreover those caused by traffic and occupational hazards, in these areas have shown an increasing trend as regards incidence, measured by mortality rates. Cardiovascular problems and malignant tumours, among the chronic diseases, reveal at times higher mortality rates than those observed in cities of highly developed countries. The incidence of traffic accidents and those due to occupational hazards is also discussed. These too are indeed more often than what is usually observed in developed countries. These aspects are increasingly demanding more services but are a problem as regards priorities for, in Latin America, infeccious diseases still contribute with very high mortality rates, on the contrary of what goes on in highly developed countries where infeccious diseases have almost been abolished. Thus, for example, in the city of S. Paulo although there is a great demand for services to cope with chronic and degenerative diseases and accident hazards, a considerable ammount of public expenditures is directed towards infeccious diseases.

UNITERMS: Urbanization. Public Health, Latin America. Chronic and degenerative diseases. Accidents. 
LALRENTI, R. - O problema das doencas cronicas e degenerativas e dos acidentes nas áreas urbanizadas da América Latina. Rev. Saícle puibl., S. Paulo, 9:239-48, 1975.

\section{REFERENCIAS BIBLIOGRAFICAS}

1. BANCO INTERAMEKICANO DO DESENVOLVIMENTO Fundo Fiduciário de Progresso Social. Progresso sócio-eco. nômico na América Latina: oitavo relatório anual, 1968. Apud: MATA, M. da et al. - Migraróes internas no Brasil: aspectos econômicos e demográficos. Rio de Janeiro, IPEA/ INPES, 1973. p. 28.

2. ESTATISTICA de Acidentes do Trabalho. CIPA - Jornal, 23(169):8-9. 1971.

3. GOLDEMBERG, P. et al. - Coeficiente de positividade das reaçōes de $\mathrm{Ma}$ chado-Guerreiro em convocados para o servico militar no Estado de $\mathrm{S}$. Paulo, de 1968 a 1971. Rev. Ass. med. bras., 20:307-8, 1974.

4. LAURENTI, R. et al. - Alguns aspectos epidemiológicos da mortalidade por acidentes de veículos a motor na cidade de São Paulo. Rev. Saúde públ., S. Paulo, 6:329-34, 1972.

5. LAURENTI, R. - Causas múltiplas de morte. São Paulo, 1973. [Tese de
Livre-Docência - Faculdade de Saúde Pública da USP]

6. PUFFER, R.R. \& GRIFFITH, G.W. Patterns of urban mortality. Washington, D.C., Pan American Health Organization, 1967. (Scient. publ. 151)

7. PLFFER, R.R. \& SERRANO, C.V. Patterns of mortality in childhood. Washington, D.C., Pan American Health Organization, 1973. (Scient. publ., 262)

8. ROMAN Y CARRILLO, G. - La epidemiologia de los accidentes del transito, Bol. Ofic. sanit. panamer., 7j: 53-65, 1973.

9. STAMLER, J. - Cardiologia preventiva. Barcelona, Ed. Científico-Médica. 1970 .

10. VINOGRADOV, N.A. - Urbanization and population's health. Hyg. Sanit., $35(1 / 3): 7-12,1970$.

Recebido para publicasão em 06-03-1975 A provado para publicação em 0.4-0.4-1.97.5 